\title{
Young stellar disks formed by the collision of a molecular cloud with a circumnuclear disk at the Galactic center
}

\author{
C. Alig ${ }^{1,2}$, M. Schartmann ${ }^{1,2}$, A. Burkert ${ }^{1,2}$ and K. Dolag ${ }^{1}$ \\ ${ }^{1}$ Universitäts-Sternwarte München, Scheinerstraße 1, D-81679 München, Germany \\ email: schartmann@mpe.mpg.de \\ ${ }^{2}$ Max-Planck-Institut für extraterrestrische Physik, Postfach 1312, Giessenbachstr., D-85741 \\ Garching, Germany
}

\begin{abstract}
We suggest a new formation mechanism for the inclined, sub-parsec scale and counterrotating stellar disks observed around the central black hole in the Milky Way Galactic center. The simulation of a single molecular cloud crashing into a circumnuclear ring of gas leads to the inflow of multiple streams of gas towards the central parsec region. The time delayed arrival of those streams forms multiple, sub-parsec scale accretion disks, with angular momentum depending on the ratio of cloud and circumnuclear ring material. These accretion disks could then be the progenitors which fragmented into the observed stellar disks. A similar event might have also led to the creation of the so-called minispiral in the Galactic center.
\end{abstract}

Keywords. black hole physics — accretion, accretion disks - hydrodynamics - methods: numerical - ISM: clouds - Galaxy: center

\section{Introduction}

The Galactic center region harbors two inclined disks of young stars at a distance of 0.05 to $0.5 \mathrm{pc}$ from Sgr A*. Their proximity to the massive black hole together with their young age of around $6 \mathrm{Myr}$ does not allow for the typical mode of star formation, as molecular clouds will be tidally sheared before they are able to form stars. A very promising model to overcome this so-called Paradox of Youth is a two-stage formation process: (i) an infalling molecular cloud will get tidally disrupted and forms an accretion disk on small scale which (ii) then fragments to form stars. Early simulations of such a process show that this results in disk and star properties in good agreement with the observed parameters, e.g. concerning the disk ellipticity, sizes, masses, etc. (Alig et al., 2011 and references therein). Furthermore, the top-heavy mass function of the stars could also be the result of fragmentation in an eccentric disk. During pericenter passage, low mass clumps will be destroyed by tidal forces and the gas will heat up, thereby increasing the Jeans mass. Both effects favor the formation of the observed top-heavy mass function of the stars in the disk (Bartko et al. 2010, Lu et al. 2013). However, in order to explain the formation of two disks, multiple such cloud encounters are necessary.

\section{Disk formation}

With the help of Gadget3 (Springel et al., 2005) simulations, we investigate a new model for the formation of the two inclined young stellar disks in the Galactic center: a collision of a single molecular cloud with a circumnuclear gas disk (similar to the one which is currently observed). Having opposite angular momentum, the interaction leads to the efficient redistribution of angular momentum and the formation of radial 

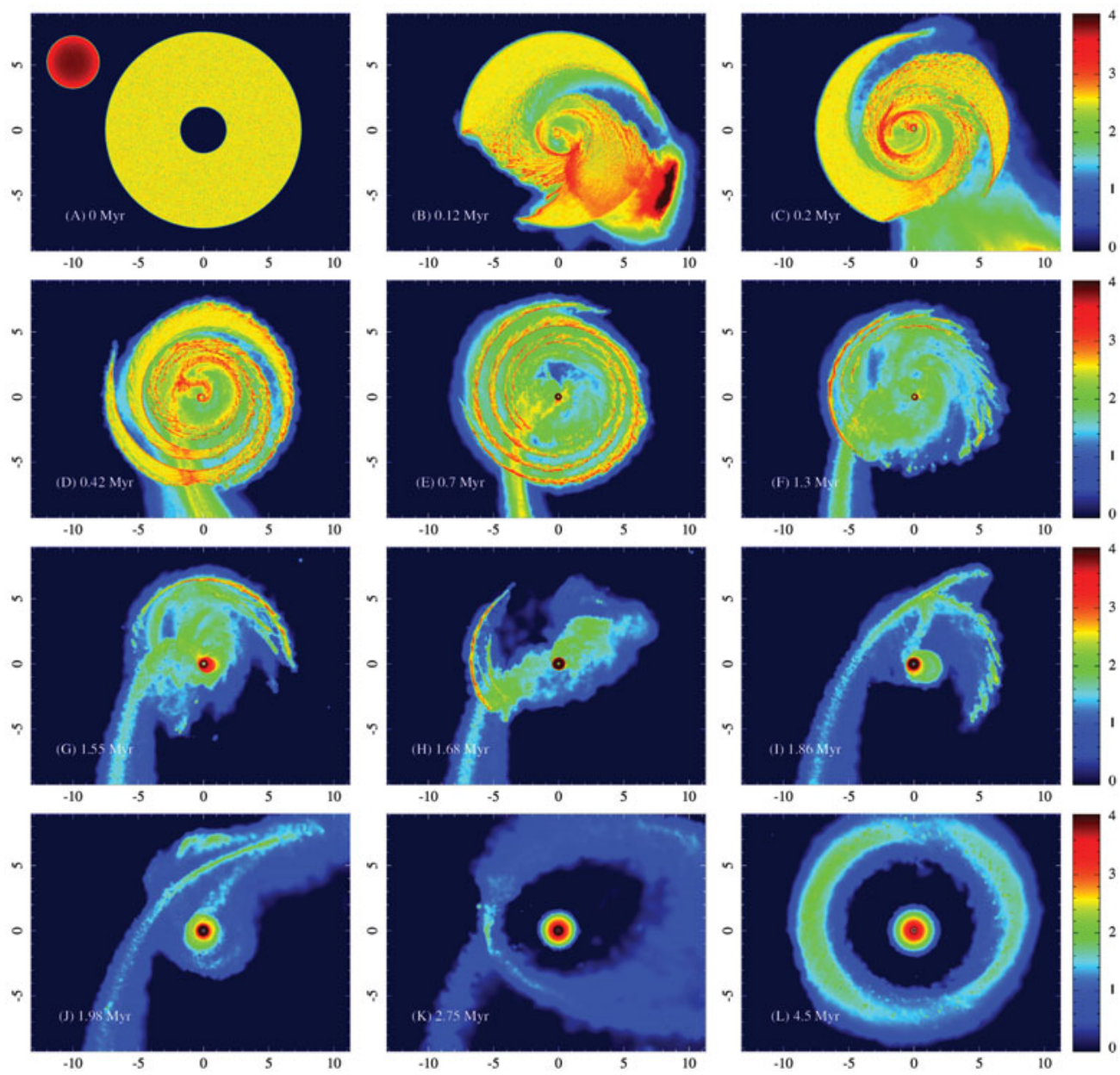

Figure 1. Density evolution of the encounter of the molecular cloud with a gas disk. Shown is the logarithmic surface density in units of $\frac{\mathrm{M}_{\odot}}{\mathrm{cm}^{-2}}$. The length units are given in pc. [A COLOR VERSiON IS AVAILABLE ONLINE.]

streams of gas, which circularize around the massive black hole (Figure 1). Depending on the mixture of cloud and circumnuclear disk gas, the forming sub-parsec scale disk will be clockwise or counter-clockwise rotating. An initial small shift of the cloud above or below the orbital plane of the gas disk even enables the formation of disks with various inclinations, resembling the clockwise and counter-clockwise rotating observed disks. The time-delayed arrival of the infalling streams enables the subsequent formation of multiple disks. Each of the disks has a lifetime of roughly 0.5 to $1 \mathrm{Myr}$, which gives the gas enough time to fragment into stars, before the next gas disk is formed, thereby destroying the previously formed disk. In summary, we presented a scenario in which a single interaction event is able to trigger the formation of the two inclined, counter-rotating disks in the GC. Previously, multiple events have been necessary, which appears much more unlikely.

A similar collision event might also be happening right now (Montero-Castaño, 2009). Depending on the parameter settings (impact parameter of the encounter, size and velocity of the cloud, etc.) the simulations also enable the formation of a three-armed spiral 
feeding the central region prior to disk-formation. Some of the latter closely resemble the so-called minispiral, which is currently observed in the parsec-scale vicinity of Sgr A*.

For more details, we refer to the original publication (Alig et al., 2013).

\section{References}

Alig, C., Burkert, A., Johansson, P. H., \& Schartmann, M. 2011, MNRAS 412, 469

Alig, C., Schartmann, M., Burkert, A., \& Dolag, K. 2013, ApJ 771, 119

Bartko, H., Martins, F., Trippe, S., Fritz, T. K., Genzel, R., Ott, T., Eisenhauer, F., Gillessen, S., Paumard, T., Alexander, T., Dodds-Eden, K., Gerhard, O., Levin, Y., Mascetti, L., Nayakshin, S., Perets, H. B., Perrin, G., Pfuhl, O., Reid, M. J., Rouan, D., Zilka, M., \& Sternberg, A. 2010, ApJ 708, 834

Lu, J. R., Do, T., Ghez, A. M., Morris, M. R., Yelda, S., \& Matthews, K. 2013, ApJ 764, 155

Montero-Castaño, M., Herrnstein, R. M., \& Ho, P. T. P.. 2009, ApJ 695, 1477

Springel, V. 2005, MNRAS 364, 1105 